\title{
A Stratification System of Ferroptosis and Iron- Metabolism Related Lncrnas Guides the Prediction of The Survival of Patients with Esophageal Squamous Cell Carcinoma
}

\section{Fangchao Zhao}

Second Hospital of Hebei Medical University

\section{Ren Niu}

Second Hospital of Hebei Medical University

\section{Yishuai Li}

Chest Hospital

\section{Zefang Dong}

Second Hospital of Hebei Medical University

\section{Xuebo Qin}

Chest Hospital

\section{Zhirong Li}

Second Hospital of Hebei Medical University

Shujun Li ( $D$ lishujun2333@163.com )

Second Hospital of Hebei Medical University https://orcid.org/0000-0001-5959-3160

\section{Primary research}

Keywords: esophageal squamous cell carcinoma, ferroptosis, IncRNA, prognostic signature, stratification system

Posted Date: November 23rd, 2021

DOl: https://doi.org/10.21203/rs.3.rs-927198/v1

License: (c) (1) This work is licensed under a Creative Commons Attribution 4.0 International License. Read Full License 


\section{Abstract}

Background: As the major type of esophageal cancer (ESCA), esophageal squamous cell carcinoma (ESCC) is also related to the highest malignant level and low survival rates across the world. Increasing people recognize long non-coding RNAs (IncRNAs) as significant mediators in regulating ferroptosis and iron-metabolism. Determining the prognostic value of ferroptosis and iron-metabolism related IncRNAs (FIRLs) in ESCC is thus critical.

Methods: Pearson's correlation analysis was carried out between ferroptosis and iron-metabolism-related genes (FIRGs) and all IncRNAs to derive the FIRLs. Based on weighted gene co-expression network exploration (WCGNA), least absolute shrinkage and selection operator (LASSO) regression and Cox regression analysis, a risk stratification system was established. According to Kaplan-Meier analysis, receiver operating characteristic (ROC) curve analysis, and univariate and multivariate Cox regression analyses, the predictive ability and clinical relevance of the risk stratification system were evaluated. The validity of the established prognostic signature was further examined in TCGA (training set) and GEO (validation set) cohorts. A nomogram with enhanced precision for forecasting OS was set up on basis of the independent prognostic elements. Gene Ontology (GO) term enrichment analysis and Kyoto Encyclopedia of Genes and Genomes (KEGG) pathway analysis were applied for the identification of pathways in which FIRLs significantly enriched. we used cell culture, transfection, CCK-8, and qRT-PCR as in vitro assays.

Results: An 3-FIRLs risk stratification system was developed by multivariate Cox regression analysis to divide patients into two risk groups. Patients in the high-risk group had worse prognosis than patients in the low-risk group. Multivariate Cox regression analysis showed the risk stratification system was an independent prognostic indicator. Receiver operating characteristic curve (ROC) analysis proved the predictive accuracy of the signature. The area under time-dependent ROC curve (AUC) reached 0.853 at 1 year, 0.802 at 2 years, 0.740 at 5 years in the training cohort and 0.712 at 1 year, 0.822 at 2 years, 0.883 at 5 years in the validation cohort. Functional enrichment analysis predicted potential associations of 49 possible upstream regulated FIRGs with ferroptosis and iron-metabolism processes and oncological signatures. Analysis of the immune cell infiltration landscape showed that ESCC in the high-risk group tended be immunologically "cold". In vitro experiments suggested that LINC01068 promoted ESCC cell proliferation.

Conclusion: The risk stratification system based on FIRLs could serve as a reliable tool for forecasting the survival of patients with ESCC.

\section{Introduction}

As a common malignant digestive system cancer, esophageal carcinoma (ESCA) was number 8 in morbidity and number 6 in mortality across the world [1]. On basis of the National Central Cancer Registry of China (NCCR) statistics, Chinese ESCA patients comprise up to $70 \%$ of all ESCA cases worldwide. 
Esophageal adenocarcinoma (EAC) and esophageal squamous cell carcinoma (ESCC) are two histopathological subtypes of ESCA. In China, $90 \%$ of patients with ESCA have ESCC [2,3]. Standardized surgery is the main treatment for esophageal cancer; however, surgery alone does not often lead to a radical cure for patients with locally advanced esophageal cancer [4]. Studies on radiotherapy and chemotherapy, targeted therapy, and biological therapy for the treatment of esophageal cancer have continued over the years; however, the 5-year survival rate of patients with esophageal cancer is less than $20 \%[5,6]$. Hence, new sensitive biomarkers for forecasting the survival of ESCC patients shall be identified as soon as possible.

Iron is elementary for the maintenance of normal roles and homeostasis in cells. Accordingly, an imbalance in iron metabolism is related to the occurrence, growth, and metastasis of cancers [7] closely. To be notable, iron metabolism plays double roles in tumor cells [8]. In the one aspect, tumor cells proliferate by more depending on iron than normal cells, which is a phenomenon of iron addiction [9]. In the other aspect, as iron concentrations increase, cell death will be caused because of accumulated reactive oxygen species and lipid peroxidation outcomes, termed ferroptosis [10,11]. As a necrotic cell death modality, Ferroptosis is varied from apoptosis, necrosis, and autophagy [12] in a morphological, biochemical, and genetical way. Recently, ferroptosis was revealed to exert various effects on biological regulation and signal transduction paths, resulting in tumor generation and progression $[13,14]$. Ferroptosis and iron metabolism have also been recognized as hidden preventive or therapeutic measures to cause cancer cell death $[15,16]$.

Long non-coding RNAs (IncRNAs) have a molecular weight greater than 200 nucleotides. Although IncRNAs account for at least $80 \%$ of the human genome, they do not take part in protein translation $[17,18]$. According to recent studies, the dysregulation of specific IncRNAs is inescapably associated with the ferroptosis process of malignant cancers[19]. Further, the upregulation of the IncRNA, NEAT1, was found to potentially regulate ferroptosis sensitivity in non-small cell lung cancer [20]. The upregulation of the IncRNA, LINC00336, was also found to inhibit ferroptosis in lung cancer by acting as a contradictive endogenous RNA [21]. Nowadays, the effect of IncRNAs on the ferroptosis process of ESCC is unknown.

In the present study, we constructed a risk stratification system, including 3 FIRLs, and systematically assessed the correlation of the risk stratification system with the prognosis and clinicopathological features of ESCC patients. Thereafter, we established a nomogram that incorporates the ferroptosis and iron-metabolism related IncRNA (FIRL) signature and clinical factors to forecast the survival of these patients. Functional enrichment analysis revealed that three FIRLs were involved in various cellular roles and signaling paths, and the immune state was varied in the high-risk and low-risk groups. In the end, the oncogenic effects of LINC01068 were explored using in vitro researches, and a new FIRL risk stratification system was developed to enhance the forecast of clinical results in patients with ESCC. To the best of our knowledge, this study firstly constructs and validates a FIRL prognostic signature for ESCC patients.

\section{Materials And Approaches}




\section{Dataset and preprocessing}

The RNA-seq transcriptome information and clinical information of ESCC patients were extracted from TCGA database (https://portal.gdc.cancer.gov/repository). LncRNAs and protein-coding genes were recognized on basis of annotation documents from the GENCODE database [22]. In addition, 296 ferroptosis and iron-metabolism related genes (FIRGs) (Table S1), including ferroptosis regulators, ferroptosis markers, ferroptosis pathway, iron uptake and transport, and iron ion homeostasis, were extracted based on previous studies [23]. The GSE53624 dataset, which includes RNA-seq information and related survival data of patients suffering from ESCC, was available from the Gene Expression Omnibus (GEO) database (http://www.ncbi.nlm.nih.gov/geo/). The multi-IncRNA prognosis model was established with the data from TCGA database as the training cohort while the predictive value of the risk score was determined with the data from GEO as the validation cohort.

\section{Identification of FIRLs}

Pearson correlation analysis was conducted using the 13,832 IncRNAs and 296 FIRGs identified $(p<$ 0.001 , correlation coefficient $>0.3$ ). Ultimately, 1,005 FIRLs were screened for follow-up bioinformatics analysis.

\section{Establishment of the weighted gene co-expression network analysis (WGCNA) network}

WGCNA is an integrated algorithm for clustering greatly related genes and identifying great modules or core genes related to a given phenotype [24]. The present research employed the WGCNA package to set up a gene co-expression network for FIRLs. Briefly, sample clustering was performed using the mean linkage approach to identify and eliminate outlier samples. Thereafter, a suitable soft thresholding power $(\beta=6)$ was selected to realize a scale-free topology fitting indicator $>0.9$. Outlier samples were eliminated using a suitable cut-off value. As the clustering performed well, a cut-line of 70 was set. Adjacency was then transformed into a topological overlap matrix (TOM) and the corresponding dissimilarity matrix (1TOM), which was applied to make the gene clustering dendrogram with a minimum module of 50 . The merging of greatly similar dynamic modules into larger modules was made at a cutline of 0.6. The associations between the modules and the immune mark were evaluated with Pearson correlation analysis. While identifying the most obvious module, the calculation of gene significance (GS) and module membership (MM) was performed. Key genes were identified as those with GS > 0.7 and $M M>$ 0.7 .

\section{Construction of the risk stratification system}

On basis of the clinical information of ESCC cases in TCGA, univariate Cox regression for FIRLs in the hub module was adopted for the identification of FIRLs associated with total survival for risk stratification system establishment. LncRNAs with a $P$ value less than 0.01 were regarded as obvious prognostic signatures. To avoid the collinearity of high-dimensional transcriptome data, the "glmnet" package was employed for least absolute shrinkage and selection operator (LASSO) regression. Finally, 
the best risk stratification system on basis of FIRLs was established using multivariate Cox regression. In particular, the risk score was determined for ESCC cases using the formula below: risk score $=$ (IncRNA 1 expression $\times$ coefficient $)+($ IncRNA 2 expression $\times$ coefficient $)+\ldots+($ IncRNA $n$ expression $\times$ coefficient $)$. According to the cut-off value of the risk score, ESCC patients in TCGA and GEO cohorts were fallen into high-risk or low-risk groups.

\section{Assessment of the clinical benefit}

Kaplan-Meier analysis and area under the ROC curves were used for the evaluation of the survival benefit, while independent prognostic factors for patients with ESCC were identified by performing univariate and multivariate Cox regression analyses. On basis of the median value of the risk score and the total survival among various groups were compared through Kaplan-Meier analysis with the log-rank test. Thereafter, the predictive precision of the FIRL signatures was evaluated by conducting a time-dependent ROC curve analysis with "survivalROC" R package. To confirm the value of the stratification system for evaluating the prognosis of ESCC patients, we combined clinical variables and performed univariate and multivariate Cox regression analyses in TCGA and GEO cohorts, respectively. To confirm the prognostic value of the stratification system for evaluating different clinical subtypes of ESCC patients, we combined patients from the GEO and TCGA cohorts into different clinical subtypes to explore the association between risk scores and clinical subgroups. A stratification system for predicting survival was also assessed using PCA, AUC, and decision curve analysis (DCA) curve to weigh the clinical practicability.

\section{Visualization of the risk stratification system}

Multivariate Cox regression analysis was adopted for the estimation of hazard ratios (HRs) and 95\% confidence intervals (Cls). "rms" R packages were employed to formulate a nomogram. The establishment of a prognostic nomogram included all independent prognostic elements recognized by multivariate Cox regression analysis to determine the potential 1-, 3-, and 5-OS of ESCC. The predictive ability of the nomogram was evaluated with AUC and calibration curve. A stratification system for predicting survival was also assessed using PCA, AUC, and DCA curve to weigh the clinical practicability.

\section{Construction of a potential regulatory network and functional analysis}

For exploring the hidden biological processes involving the 3 FIRLs, we identified 49 possible upstream regulated FIRGs through Pearson correlation analysis. Thereafter, gene enrichment analysis was performed with differentially expressed FIRGs using "ggplot2" and "clusterProfiler" packages in R software.

\section{Immune landscape analysis}

We used the ssGSEA algorithm [25] ('GSVA' package in R software) to conduct immune landscape analysis, which mainly included correlations between 24 immune cells and immune-related functions. 


\section{Vitro assays}

In this study, we used cell culture, transfection, CCK-8, and qRT-PCR as in vitro assays. The Shanghai Cell Institute Country Cell Bank provided normal and ESCC cell lines. GenePharma generated and annealed small-interfering RNA (si-RNA-1/2/3) oligos for LINC01068 and a general negative control. Following the manufacturer's procedure, the transfection of each siRNA duplex into cells was made with Lipofectamine ${ }^{\circledR} 2000$ (Invitrogen, Carlsbad, CA, USA). Details of these methods are provided elsewhere [26].

\section{Results}

\section{Identification of core FIRLs in ESCC patients by Pearson and WCGNA}

Based on previous literature, we collected 296 FIRGs (Table S1). For TCGA cohort, Pearson correlation analysis was performed using the 296 genes and all annotated IncRNAs. Ultimately, 1005 FIRLs were we identified (Figure 1A). The clustering of each sample was good, and only one outlier sample was eliminated (the cutting line was 500). Topological calculation was then performed with a soft threshold value of 1 to 20, and an optimal soft threshold value of 4 (Figure 1B). According to the soft threshold, the relationship matrix was finally converted into a TOM, and the related modules were classified according to the TOM. The number of genes in each module was not less than 50 , and the shear height of gene modules was 0.6 (Figure 1C). By using Pearson correlation analysis to decide the correlation between the modules and clinical traits, seven modules were identified. Of note, the blue module had the strongest correlation with pathological staging and survival status. Accordingly, this module was recognized as the core module in ESCC patients (Figure 1D). Finally, the 151 FIRLs in the module were found to be associated with the occurrence of ESCC closely (Table S2).

\section{Construction of a risk stratification system}

To identify survival-related FIRLs, univariate Cox analyses were performed using $151 \mathrm{FIRLs}$ in the blue module. Finally, 16 FIRLs were screened for subsequent analyses (Figure 2A). For further decreasing the number of genes in the signature, the subjection of 16 FIRLs to LASSO regression analysis was performed (Figure 2B, C). Thereafter, three FIRLs from LASSO were retrieved and subjected to multivariate Cox regression analysis to develop a risk stratification system (Figure 2D; Table 1). The calculation of the risk score of ESCC patients was made below: risk score $=0.5697 \times$ LINC01068 $+0.5154 \times$ TMEM92-AS1 $+0.5964 \times$ AC243967.2). 
Table 1

The 3-LnCRNA were identified by Cox and LASSO regression analysis

\begin{tabular}{|llllll|}
\hline LncRNA & coef & HR & HR.95L & HR.95H & P-value \\
\hline LINC01068 & 0.5697 & 1.7678 & 1.1665 & 2.6788 & 0.0072 \\
\hline TMEM92-AS1 & 0.5154 & 1.6743 & 0.9593 & 2.9223 & 0.0697 \\
\hline AC243967.2 & 0.5964 & 1.8157 & 1.0883 & 3.0292 & 0.0224 \\
\hline
\end{tabular}

\section{Clinical benefits of the risk stratification system}

On basis of the cut-off value of risk scores, ESCC patients from the TCGA cohort were divided into two risk groups: high-risk $(n=40)$ and low-risk $(n=40)$. Using the same cut-off value, ESCC patients in the GEO cohort were fallen into high-risk $(n=31)$ and low-risk $(n=88)$ groups. As shown in Figure 3E, the AUCs of the 3 FIRL risk stratification system performed with TCGA cohort were $0.712,0.822$, and 0.883 at 1,3 , and 5 years. In addition, the 1-year survival prediction in the GEO cohort showed good results (Figure $3 A)$. The association between the risk mark and prognosis of ESCC patients was explored with the Kaplan-Meier method and log-rank tests. Patients in the high-risk group were found to have a lower survival rate than those in the low-risk group $(P<0.001)$ (Figure 3B, F). Furthermore, in the prediction of median survival time, ROC curve analysis revealed that the risk mark showed better predictive performance than the other clinicopathological features (Figures 3C, G). DCA also suggested that in actual clinical applications, this risk score had a better value than traditional pathological staging (Figures 3D, H).

\section{Risk stratification system is an independent prognostic element for ESCC patients}

For determining whether the risk score was an independent prognostic element for ESCC patients, univariate and multivariate Cox regression analyses were conducted using the clinical features and risk score. Based on the outcomes of univariate Cox regression analysis, the risk score was greatly related to OS in both TCGA and GEO cohorts (TCGA cohort: HR $=2.769,95 \% \mathrm{Cl}=1.175-7.866, p=0.036$; GEO cohort: $\mathrm{HR}=1.443,95 \% \mathrm{Cl} 1.143-1.821, p=0.002$ ) (Figures 4A, C). After the modification for other confounders, the risk score was still an independent predictor of OS in multivariate Cox regression analysis (TCGA cohort: $\mathrm{HR}=3.750,95 \% \mathrm{Cl}=1.151-12.219, p=0.028 ; \mathrm{GEO}$ cohort: $\mathrm{HR}=1.242,95 \% \mathrm{Cl}=$ $1.115-1.687, p=0.025$; Figures 4B, D).

\section{Subgroup analysis of the risk stratification system in the total cohort}

To determine the prognostic value of the risk stratification system for ESCC patients based on different clinical characteristics, subgroups were derived on basis of age ( $\leq 65 \mathrm{vs.}>65$ years), sex (male vs. female), clinical phase (I-II vs. III-IV), T phase (T0-T2 vs. T3-T4), and N phase (N0 vs. N1-N3). 
Accordingly, the stratification system was found to have a better predictive value for NO, I-II, and male patients, which may indicate that the risk stratification system showed better predictive value for patients with early stage ESCC (Figures 5A-J).

\section{Survival analysis and clinical correlation analysis of FIRLs in the risk stratification system}

For further exploring the association between the risk stratification system and clinical parameters, we constructed two composite heat maps for patients from TCGA (Figure 6A) and GEO (Figure 6E) cohorts. A heat map could display the risk scores, clinicopathological parameters, and FIRL expression for each group. A survival analysis of FIRLs participating in the risk stratification system was also performed. Based on the results, AC243967.2 and LINC01068 were identified as high-risk factors for ESCC patients (high expression of AC243967.2 and LINC01068 was related to poor survival rate of ESCC patients) ( $P<$ 0.05 ) in TCGA cohort (Figures 6B-D). However, LINC01068 was suggested to have a better predictive value $(P<0.05)$ in the GEO cohort (Figures $6 \mathrm{~F}-\mathrm{H})$. For further exploring the association between different risk groups and clinical characteristics, we merged all patients in the GEO and TCGA datasets and analyzed the differences in risks of different pathological characteristics based on the available clinical information. According to Figures 6I-M, no disparity in risk scores was found according to gender, age, $\mathrm{N}$ stage, and clinical stage. However, with respect to $T$ stage, risk scores were greatly varied between the groups.

\section{Construction and verification of a nomogram based on the risk stratification system}

The OS of patients with ESCC was predicted by establishing a nomogram on basis of independent predictive elements originated from a multivariate Cox risk regression model (Figures 7A). According to the prediction model calibration curve, consistent predicted and actual survival rates for the training and validation sets were revealed (Figures 7B-H).

\section{Regulatory network of the potential biological functions of 3 FIRLS}

To explore the potential biological processes involving the three FIRLs, 49 possible upstream regulated FIRGs were identified through Pearson correlation analysis (Figures 8A). The analyses of GO functional enrichment and KEGG pathway enrichment were conducted on the 49 FIRGs. On basis of the outcomes of KEGG analysis, in addition to ferroptosis, 49 FIRGs were mainly enriched in the IL-17 signaling pathway, HIF-1 signaling pathway, VEGF signaling pathway, and TNF signaling pathway (Figures 8B). Further, GO analysis results indicated that in addition to iron death, iron metabolism, and other related processes, the 49 FIRGs were related to DNA damage response and signal transduction by p53 class mediator processes (Figures 8C-E).

Immunity Analyses 
For further exploring the associations between the risk scores and immune cells and functions, the enrichment scores of 16 immune cell subpopulations and their associated functions were quantified with the ssGSEA R package. The kinds of immune cells (including DCs, iDCs, mast cells, NK cells, pDCs, T helper cells, Tfh cells, and Th1 cells) in the high-risk group were found to greatly differ from those in the low-risk group (Figures 9A, C). In addition, the scores of the immune functions, such as the CCR, check point, HLA, parainflammation, T cell co-inhibition, T cell co-stimulation, and Type II IFN response, were greatly higher in the low-risk group than in the high-risk group, indicating that their immunological functions related to FIRLs were more active in the low-risk group (Figures 9B, D).

\section{In vitro assays for validation}

To further validate the bioinformatics results, the expression level of LINC01068 mRNA in ESCC cell lines was detected. It was found that the expression of LINC01068 is upregulated in ESCC cell lines by comparing with the normal cell line, as shown in Figure 10A. In addition, si-LINC01068 and si-NC were transfected into Eca109 and TE-1 cells, respectively, and qRT-PCR was adopted for the detection of the expression of LINC01068. LINC01068 expression was downregulated in ESCC cell lines after transfection, as shown in Figure 10B and D. Similarly, the CCK-8 assays revealed that ESCC cell proliferation was inhibited after transfection with LINC01068, as shown in Figure 10C and E.

\section{Discussion}

The transformation of next-generation sequencing has been performed for prognosis of cancer. In clinical routines, the prognosis of cancer patients cannot be adequately predicted with the conventional staging system. Biomarkers associated with tumor diagnosis and prognosis are thus urgently needed $[27,28]$.

Due to the disturbances in iron metabolism, overmuch intracellular iron storage was caused with ferroptosis induced [29]. As a hallmark of tumors, Ferroptosis is greatly related to the prognosis of cancer patients [30]. Because of the significant effect of ferroptosis and iron metabolism on cancer, remarkable attention has been paid to its associated IncRNAs [31].

To the best of our knowledge, this research firstly identifies and analyzes prognostic FIRLs in ESCC in a comprehensive way. On basis of previous studies, we collected 296 FIRGs. In TCGA cohort, Pearson correlation analysis of 296 genes and all annotated IncRNAs was performed, and 1005 FIRLs were identified. Through WGCNA, 151 core FIRLs were identified in the blue module, and a risk stratification system comprising 3 FIRLs (LINC01068, TMEM92-AS1, and AC243967.2) was established by integrating LASSO regression and Cox regression analyses. The assignment of all patients to high- and low-risk groups was performed on basis of risk scores. On basis of Kaplan-Meier curve analysis, high-risk groups were related to dismal OS by comparing with low-risk groups. The ROC curve indicates the excellent performance of our risk stratification system. The AUCs of the ROC plots for one-, three-, and five-year OS in TCGA cohort were $0.712,0.822$, and 0.883 . In addition, the stratification system for predicting survival was assessed by PCA, AUC, and DCA curve to weigh the clinical practicability. Based on the results, our risk signature consistently realized good predictive value by comparing with other risk prognostic 
signatures published for ESCC. The GEO cohort was adopted to verify the established prognostic signature. Moreover, other clinicopathological features and prognostic signatures were combined for Cox analysis, which ultimately verified that the constructed risk stratification system may be used as an independent prognostic element for ESCC patients. Herein, while establishing a nomogram, whether the nomogram was precise at predicting one-, three-, and five-year OS was determined with calibration plots. Altogether, our findings indicate that the risk stratification system could be a high-class predictor relative to the conventional clinical indicator.

To determine the potential biological processes involving the 3 FIRLs, we identified 49 possible upstream regulated FIRGs through Pearson correlation analysis, and further conducted functional enrichment analysis with these genes to dig the potential biological pathways.

The role of immunotherapy relies on the immunogenicity of the tumor immune microenvironment (TIME). Therefore, knowing TIME is core for the evaluation of immunotherapy [32]. Based on immune infiltration analysis, the abundance of immune cells (DCs, iDCs, mast cells, NK cells, pDCs, T helper cells, Tfh, Th1 cells, and TIL cells) in the low-risk group was higher than that in the high-risk group. Further, several immune system process pathways were found to be participated in, indicating that low-risk patients have higher immunogenicity in TIME and exhibit better answer to immunotherapy. The outcomes of our research may thus assist in guiding immunotherapy for ESCC.

In the end, the association between LINC01068 and ESCC progression was determined. The inhibition of LINC01068 inhibited the cell viability and migration of Eca109 and TE-1 cells, which further verified the carcinogenic effect of LINC01068 on digestive system neoplasms.

This study had some limitations. First, the FIRL risk stratification system was constructed and validated using a public database. However, the use of prospective, multicenter, real-world data for the assessment of the clinical utility of this system would be more ideal. Second, the association between FIRLs and antitumor immunity was preliminarily revealed by our research. Therefore, it is necessary to further dig the hidden mechanisms. Finally, the signaling pathways involved in FIRLs were only preliminarily explored. Accordingly, the specific mechanism of FIRLs in ESCC and their association with ferroptosis are not completely acknowledged. More studies are thus needed to validate our findings.

In summary, this study fills a gap regarding the use of FIRLs for the prognostic forecast of ESCC. The prognostic FIRLs derived in our research displayed robust capacity at forecasting the survival results of ESCC patients and were related to the immune landscape of the ESCC microenvironment. The risk stratification system based on FIRLs could serve as a reliable tool for forecasting the survival of patients with ESCC.

\section{Declarations}

\section{Ethics approval and consent to participate}


Not applicable

\section{Consent for publication}

We have approved the manuscript and agreed to publish it.

\section{Availability of data and materials}

Publicly available datasets were analyzed in this study. These data can be found here: TCGA (https://portal.gdc.cancer.gov/), GEO database (https://www.ncbi.nlm.nih.gov/geo/).

\section{Competing interests}

The authors declare that they have no competing interests.

\section{Funding}

The authors received no funding for this work.

\section{Authors' contributions}

FCZ conceived the study and drafted the manuscript; YSL, ZFD, XBQ and ZRL collected the data; RN confirmed and sorted out the data; SJL revised this manuscript. All authors read and approved the final manuscript.

\section{Acknowledgements}

Not applicable.

\section{References}

[1] Gupta B, Kumar N. Worldwide Incidence, Mortality and Time Trends for Cancer of the Oesophagus. Eur J Cancer Prev. 2017;26(2):107-18.

[2] Yang YM, Hong P, Xu WW, et al. Advances in targeted therapy for esophageal cancer. Signal Transduct Target Ther. 2020;5:229.

[3] Abnet CC, Arnold M, Wei WQ. Epidemiology of Esophageal Squamous Cell Carcinoma. Gastroenterology. 2018;154(2):360-73.

[4] Imazeki H, Kato K. Development of chemotherapeutics for unresectable advanced esophageal cancer. Expert Rev Anticancer Ther. 2020;20:1083-1092.

[5] Yang J, Liu X, Cao S, et al. Understanding Esophageal Cancer: The Challenges and Opportunities for the Next Decade. Front Oncol. 2020;10:1727. 
[6] Huang FL, Yu SJ. Esophageal cancer: Risk factors, genetic association, and treatment. Asian J Surg. 2018;41:210-215.

[7] Yang WS, SriRamaratnam R, Welsch ME, et al. Regulation of ferroptotic cancer cell death by GPX4. Cell. 2014;156:317-331.

[8] Wang Y, Yu L, Ding J, et al. Iron Metabolism in Cancer. Int J Mol Sci. 2018;20(1):95.

[9] Basuli D, Tesfay L, Deng Z, et al. Iron addiction: a novel therapeutic target in ovarian cancer. Oncogene. 2017;36(29):4089-4099.

[10] Chen X, Yu C, Kang R, et al. Iron metabolism in ferroptosis. Front Cell Dev Biol. 2020;8:590226.

[11] Battaglia AM, Chirillo R, Aversa I, et al. Ferroptosis and cancer: mitochondria meet the "Iron Maiden" cell death. Cells. 2020;9:1505.

[12] Dixon SJ, Lemberg KM, Lamprecht MR, et al. Ferroptosis: an iron-dependent form of nonapoptotic cell death. Cell. 2012;149:1060-1072.

[13] Stockwell BR, Friedmann Angeli JP, Bayir H, et al. Ferroptosis: a regulated cell death nexus linking metabolism, redox biology, and disease. Cell. 2017;171(2):273-285.

[14] Xu T, Ding W, Ji X, et al. Molecular mechanisms of ferroptosis and its role in cancer therapy. J Cell Mol Med. 2019;23(8):4900-4912.

[15] Shen Z, Song J, Yung BC, et al. Emerging Strategies of Cancer Therapy Based on Ferroptosis. Adv Mater. 2018;30:e1704007.

[16] Tang B, Zhu J, Li J, et al. The ferroptosis and iron-metabolism signature robustly predicts clinical diagnosis, prognosis and immune microenvironment for hepatocellular carcinoma. Cell Commun Signal. 2020;18(1):174.

[17] Yu J, Wu X, Huang K, et al. Bioinformatics identification of IncRNA biomarkers associated with the progression of esophageal squamous cell carcinoma. Mol Med Rep. 2019; 19:5309-20.

[18] Statello L, Guo CJ, Chen LL, et al. Gene regulation by long non-coding RNAs and its biological functions. Nature Reviews Molecular Cell Biology. 2021;22(2):96-118.

[19] Schmitt AM, Chang HY. Long Noncoding RNAs in Cancer Pathways. Cancer Cell. 2016;29(4):452-463.

[20] Wu H, Liu A. Long non-coding RNA NEAT1 regulates ferroptosis sensitivity in non-small-cell lung cancer. J Int Med Res. 2021;49(3):300060521996183.

[21] Wang M, Mao C, Ouyang L, et al. Long noncoding RNA LINC00336 inhibits ferroptosis in lung cancer by functioning as a competing endogenous RNA. Cell Death Differ. 2019;26(11):2329-2343. 
[22] Harrow J, Frankish A, Gonzalez JM, et al. GENCODE: the reference human genome annotation for The ENCODE Project. Genome Res. 2012;22(9):1760-1774.

[23] Yao J, Chen X, Liu X, et al. Characterization of a ferroptosis and iron-metabolism related IncRNA signature in lung adenocarcinoma. Cancer Cell Int. 2021;21(1):340.

[24] Pei G, Chen L, Zhang W. WGCNA Application to Proteomic and Metabolomic Data Analysis. Methods Enzymol. 2017;585:135-158.

[25] Hänzelmann S, Castelo R, Guinney J. GSVA: gene set variation analysis for microarray and RNA-seq data. BMC Bioinformatics. 2013;14:7.

[26] Li X, Ma TK, Wen S, et al. LncRNA ARAP1-AS2 promotes high glucose-induced human proximal tubular cell injury via persistent transactivation of the EGFR by interacting with ARAP1. J Cell Mol Med. 2020;24(22):12994-13009.

[27] Su H, Hu N, Yang HH, et al. Global gene expression profiling and validation in esophageal squamous cell carcinoma and its association with clinical phenotypes. Clin Cancer Res. 2011;17(9):2955-66.

[28] Li J, Chen Z, Tian L, et al. LncRNA profile study reveals a three-IncRNA signature associated with the survival of patients with oesophageal squamous cell carcinoma. Gut. 2014;63:1700-1710.

[29] Bogdan AR, Miyazawa M, Hashimoto K, et al. Regulators of iron homeostasis: new players in metabolism, cell death, and disease. Trends Biochem Sci. 2016;41(3):274-286.

[30] Chen X, Kang R, Kroemer G, et al. Broadening Horizons: The Role of Ferroptosis in Cancer. Nat Rev Clin Oncol. 2021;18(5):280-96.

[31] Mou Y, Wang J, Wu J, et al. Ferroptosis, a new form of cell death: opportunities and challenges in cancer. J Hematol Oncol. 2019;12(1):34.

[32] Gentles AJ, Newman AM, Liu CL, et al. The prognostic landscape of genes and infiltrating immune cells across human cancers. Nat Med. 2015;21:938-945.

\section{Figures}


A

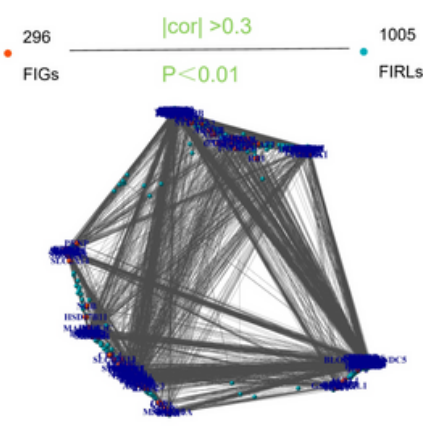

C

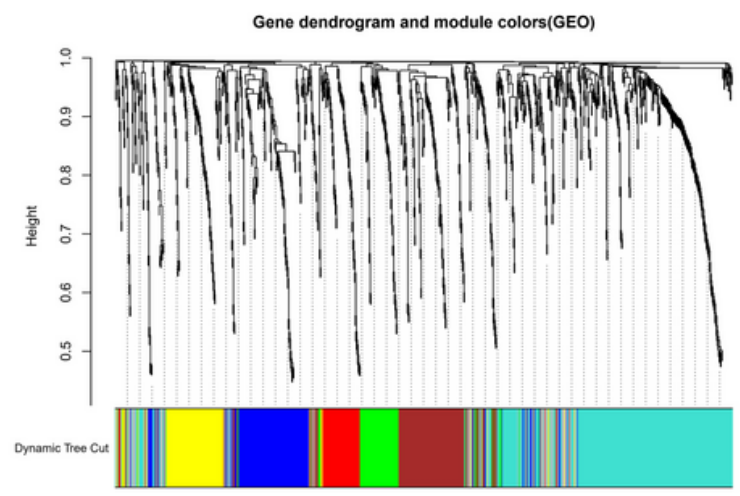

$\mathrm{B}$
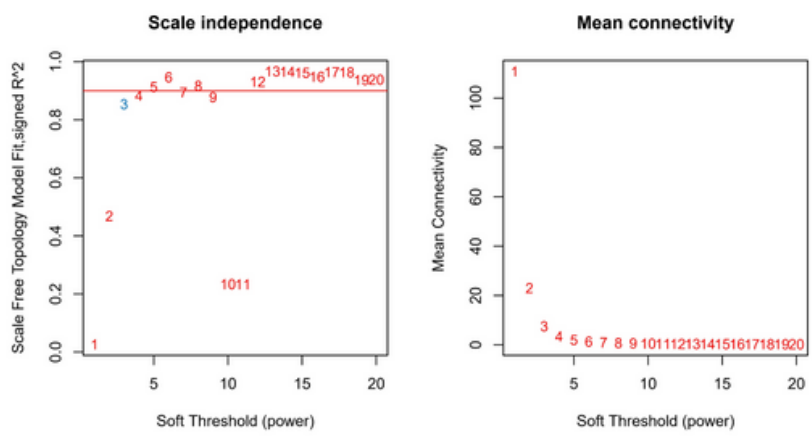

D

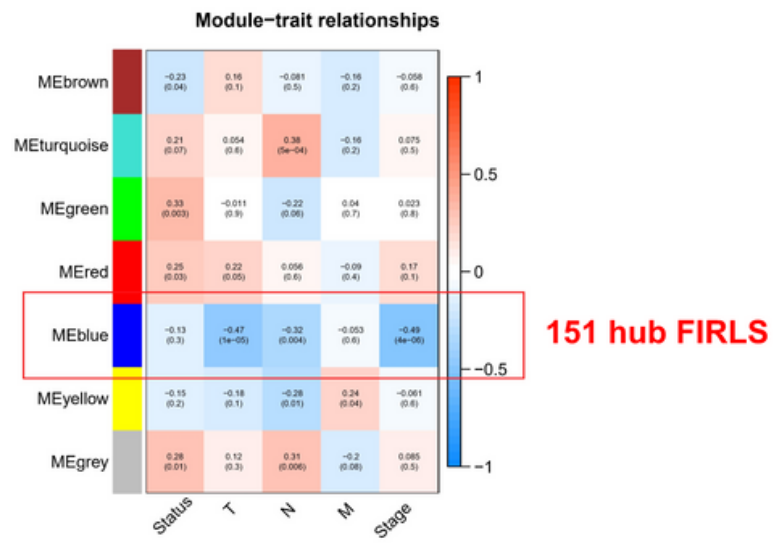

\section{Figure 1}

Screening of survival-related IncRNAs by weighted gene co-expression network analysis (WGCNA). (A) Identification of ferroptosis and iron-metabolism-related IncRNAs (FIRLs) using Pearson correlation analysis. (B) Soft power in WGCNA. (C) Clustering and merging of the co-expression modules. (D) Association heatmap of module genes and clinical features. Red means positive association, and blue refers to negative association. Correlation grows as the color darkens. 
A

16 hub prognostic FIRLS

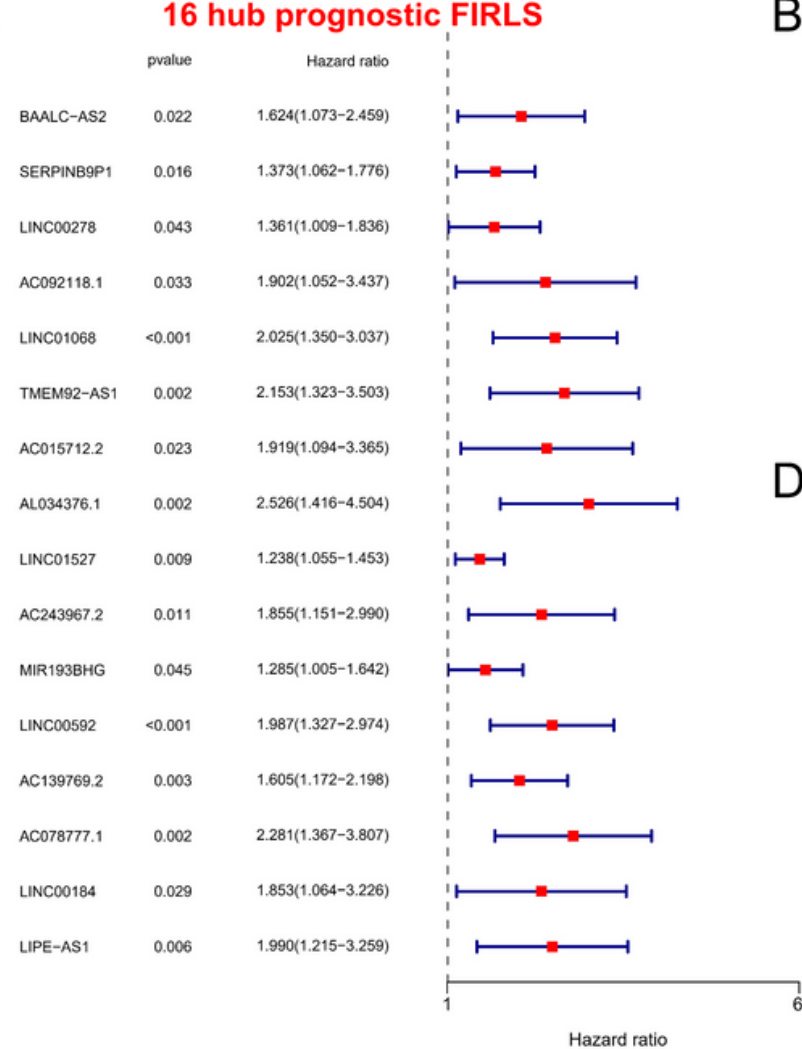

B

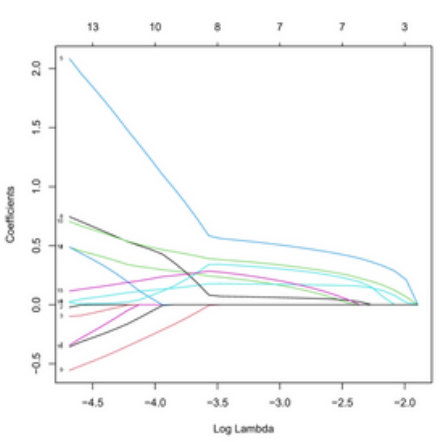

D

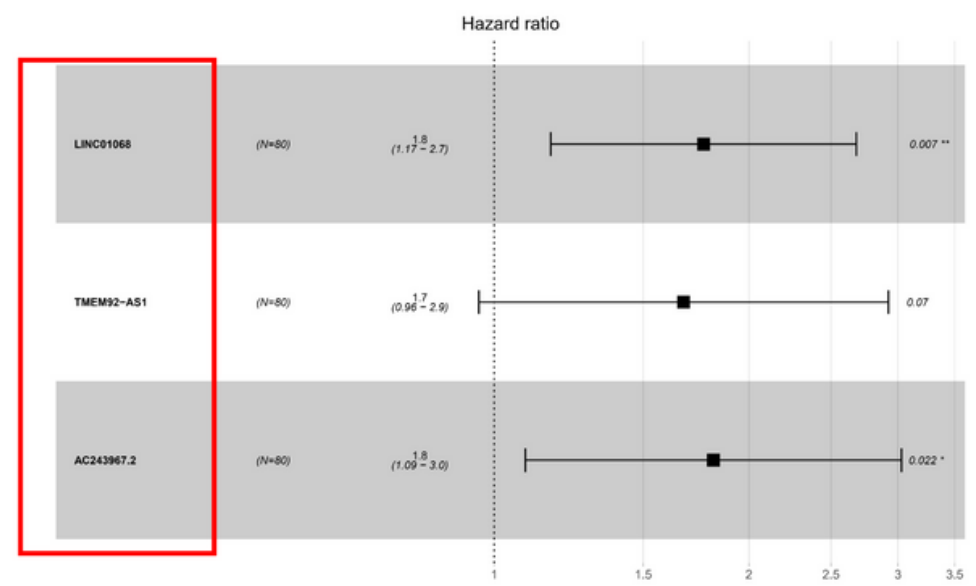

\section{Figure 2}

Construction of the risk stratification system. (A) T he ferroptosis and iron-metabolism-related IncRNAs (FIRLs) that significantly correlated with survival were identified by univariate analysis. (B, C) LASSOvalidation. (D) Forest plot of hazard ratios showing the prognostic value of the 3 FIRLs. 

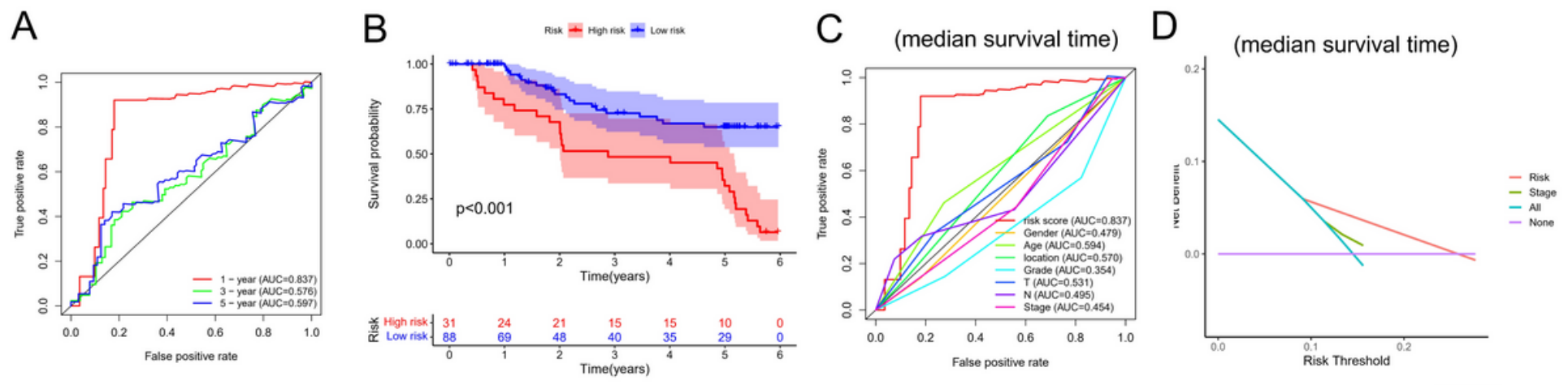

GEO cohort
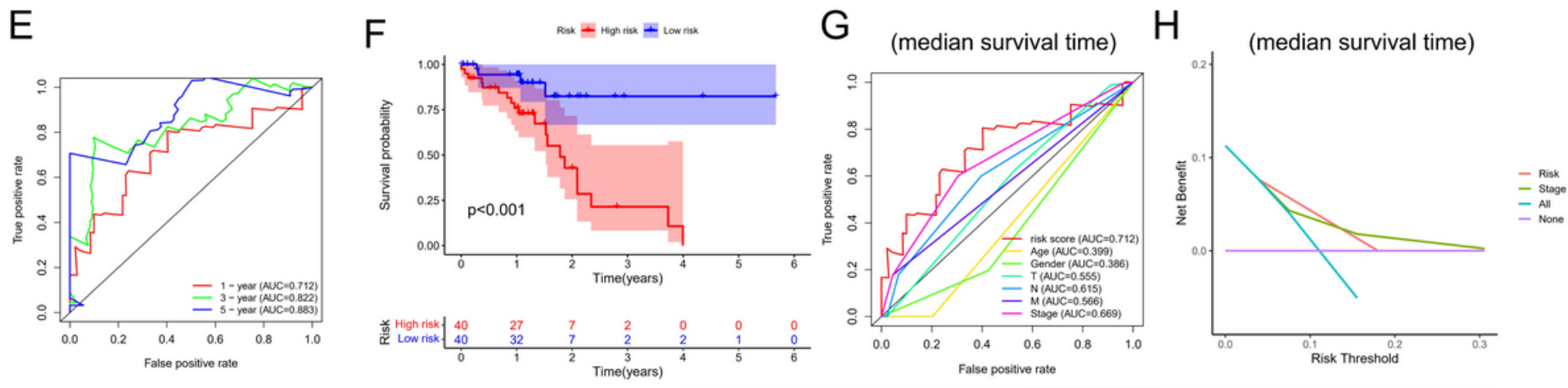

TCGA cohort

\section{Figure 3}

Clinical benefits of the risk stratification system. (A, E) Time-dependent ROC curves. (B, F) Kaplan-Meier analysis of high- and low-risk patients. $(C, G)$ ROC curve analysis revealed the prognostic accuracy of risk mark and clinicopathological coefficients. (D, H) Decision curve analysis (DCA). 
A

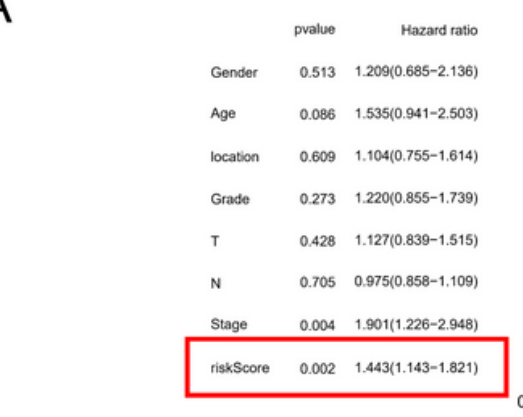

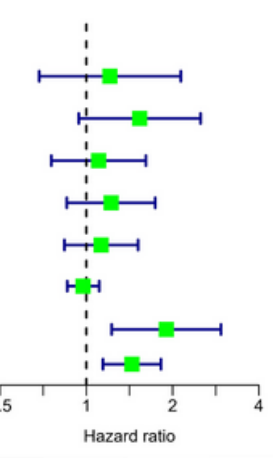

B

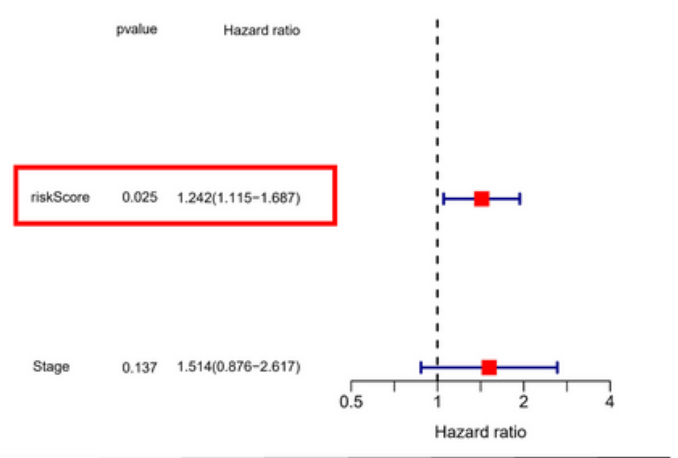

C

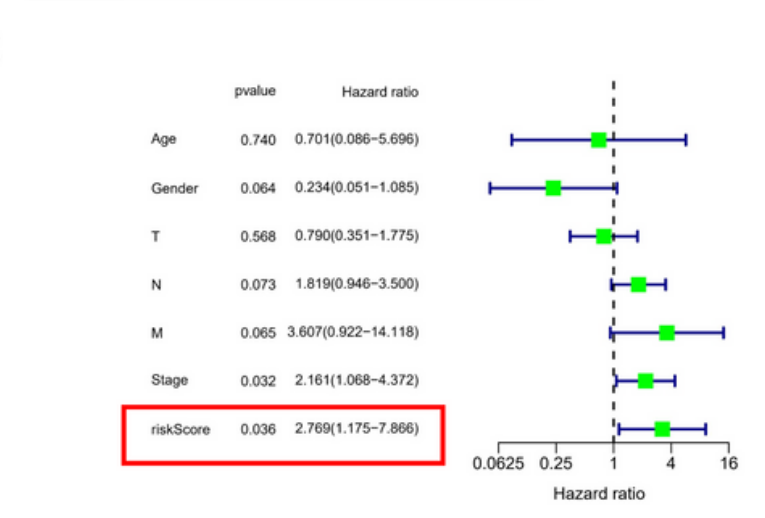

GEO cohort

D

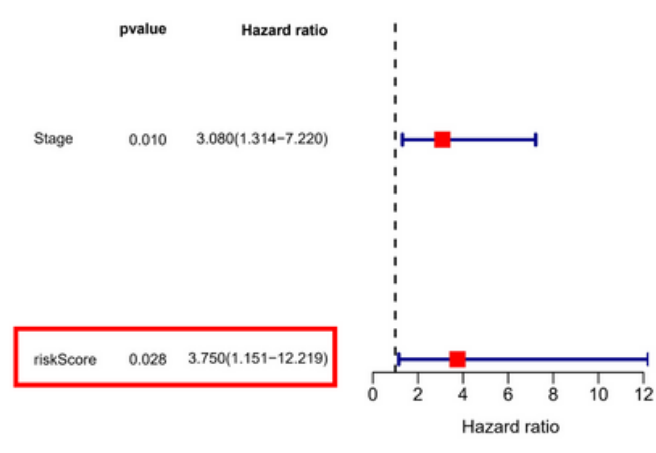

TCGA cohort

\section{Figure 4}

Evaluation of the prognostic values of risk stratification. (A, B) Univariate and multivariate Cox regression analyses of the risk scores in GEO. (C, D) Univariate and multivariate Cox regression analyses of the risk scores in TCGA. 

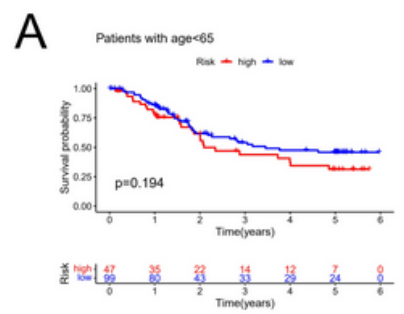

B

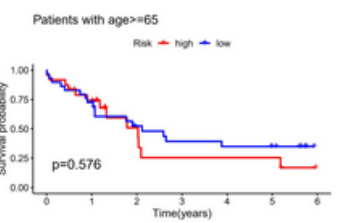

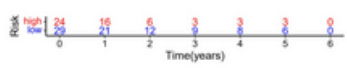

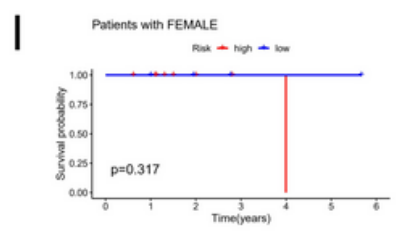

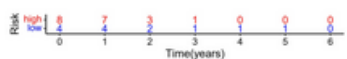

C

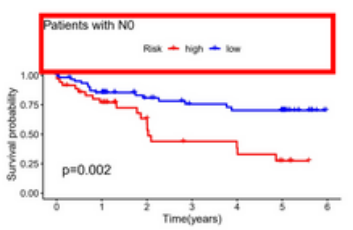

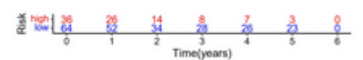

$\mathrm{D}$

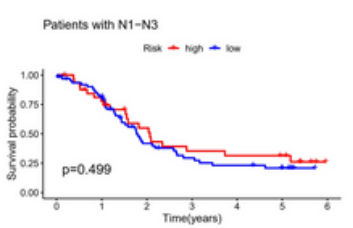

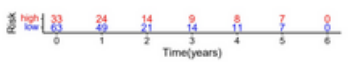

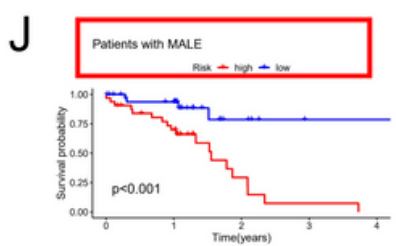

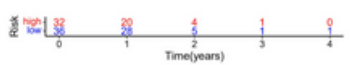

$\mathrm{E}$

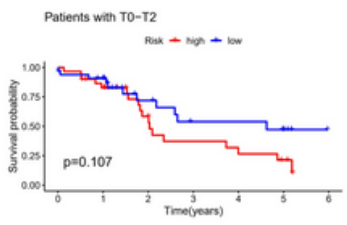

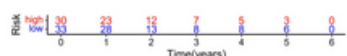

$\mathrm{F}$

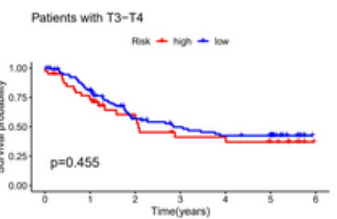

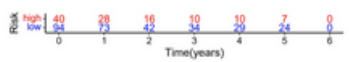

G

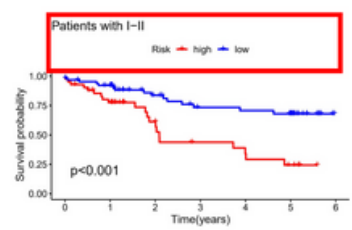

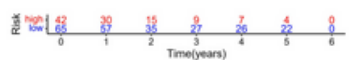

$\mathrm{H}$

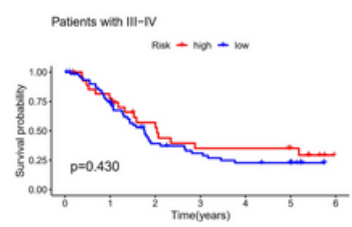

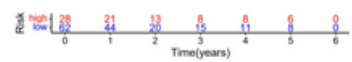

\section{Figure 5}

Subgroup analysis based on different clinical characteristics of the risk stratification system. $(A)$ age $\leq$ 65, (B) age > 65, (C) NO, (D) N1-N3, (E) T0-T2, (F) T3-T4, (G) stage I-II, (H) stage III- $\mathrm{B}$, (I) female, and (J) male. 

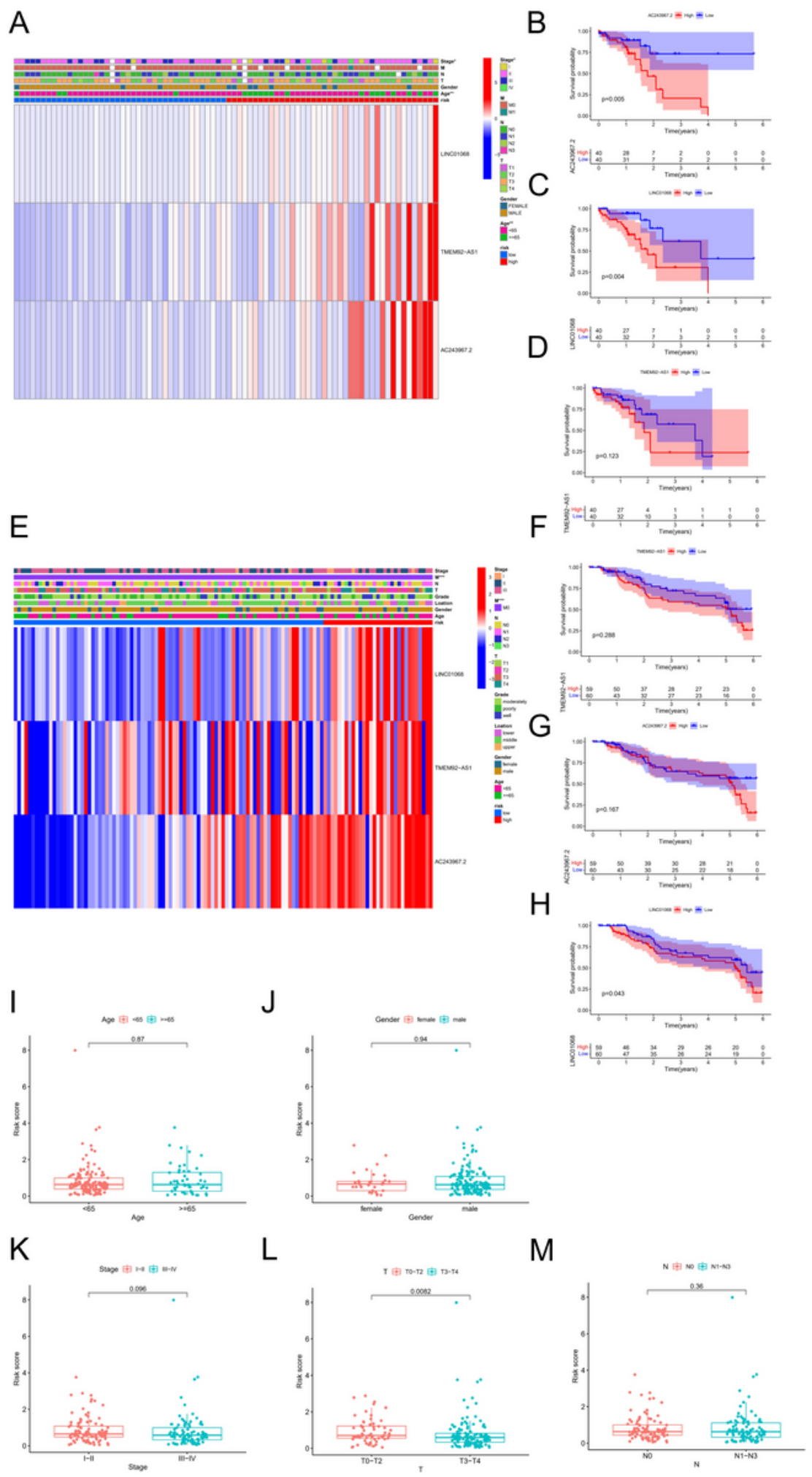

\section{Figure 6}

Survival analysis and clinical correlation analysis of ferroptosis and iron-metabolism-related IncRNAs (FIRLs) in risk stratification. (A) A composite heat map containing clinical information and expression of 3 FIRLs in TCGA cohort. (B-D) Survival analysis of FIRLs participating in risk stratification in TCGA cohort. (E) A composite heat map containing clinical information and expression of 3 FIRLs in the GEO cohort. 
(F-H) Survival analysis of FIRLs participating in risk stratification in the GEO cohort. (I-M) Box plot of correlation analysis between risk grouping and clinical information in the entire cohort.

A
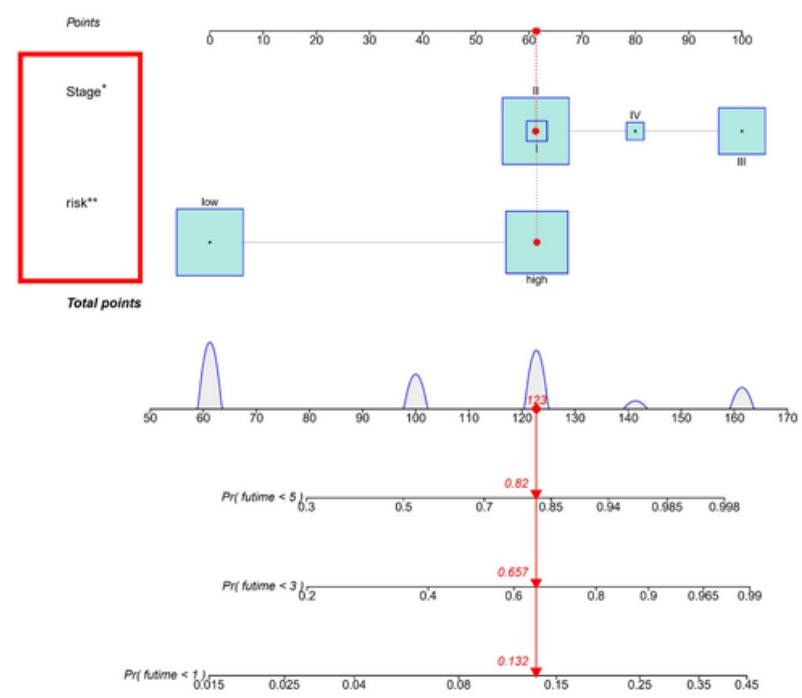

B

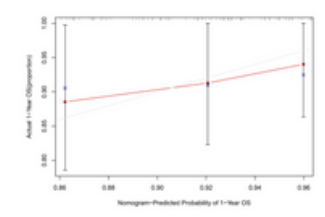

C

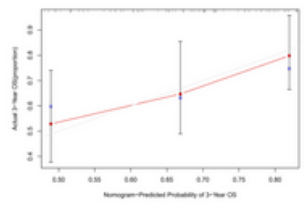

GEO cohort

E

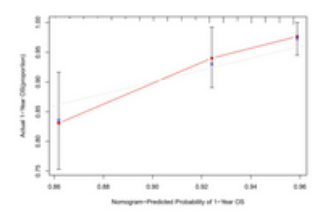

$\mathrm{F}$

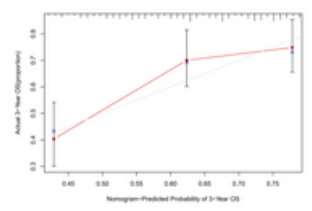

TCGA cohort
$\mathrm{H}$

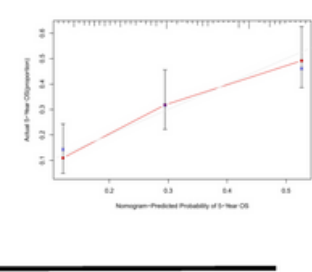

D

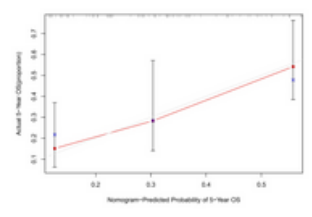

(1)

\section{Figure 7}

Establishment and verification of a nomogram. (A) Nomogram for forecasting the total survival (OS) of patients with esophageal squamous cell carcinoma (ESCC) at 1, 3, and 5 years. Calibration curves of nomogram for OS forecast at 1, 3, and 5 years in (B-D) TCGA and (E-G) GEO cohorts. 
A

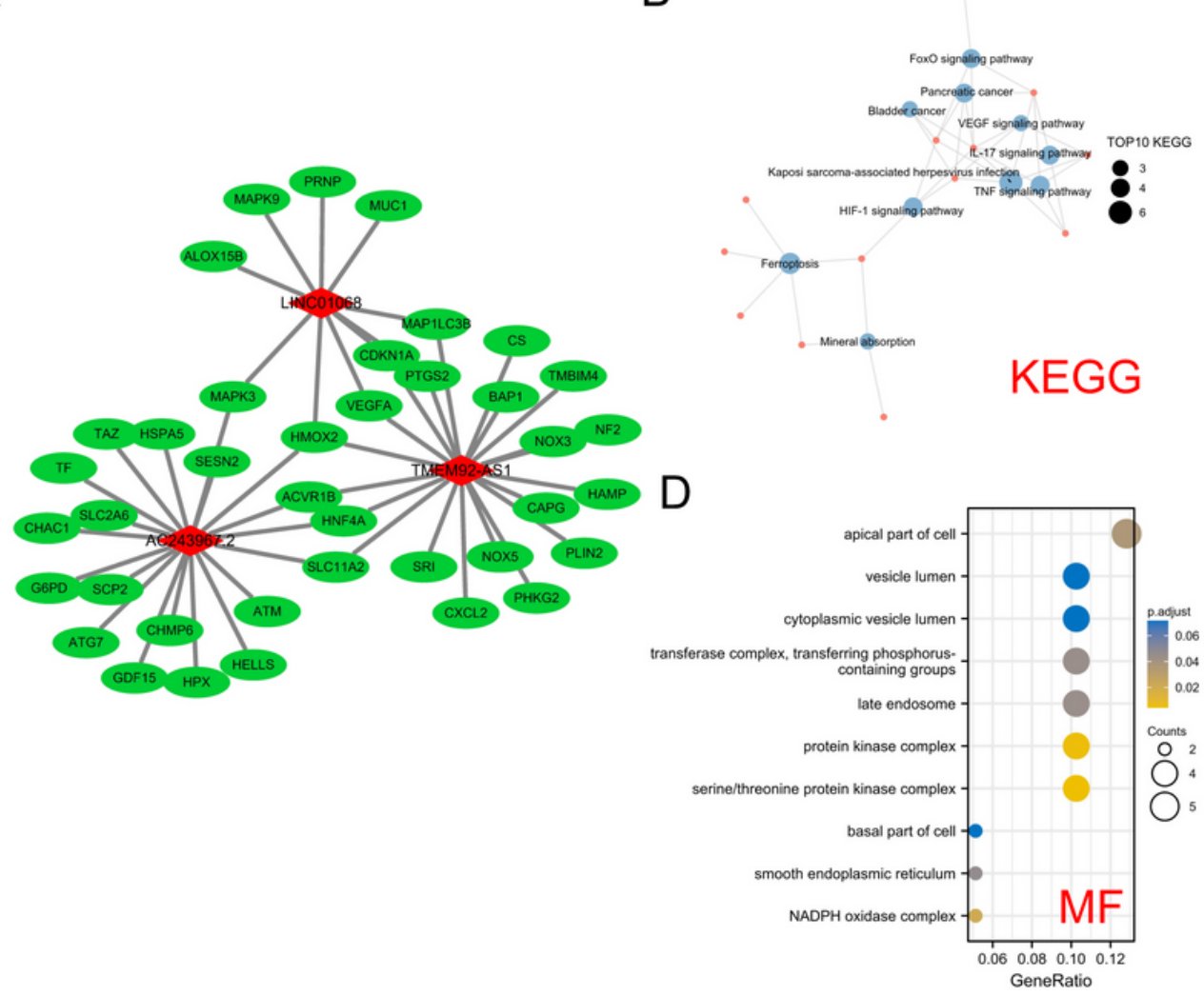

B
C

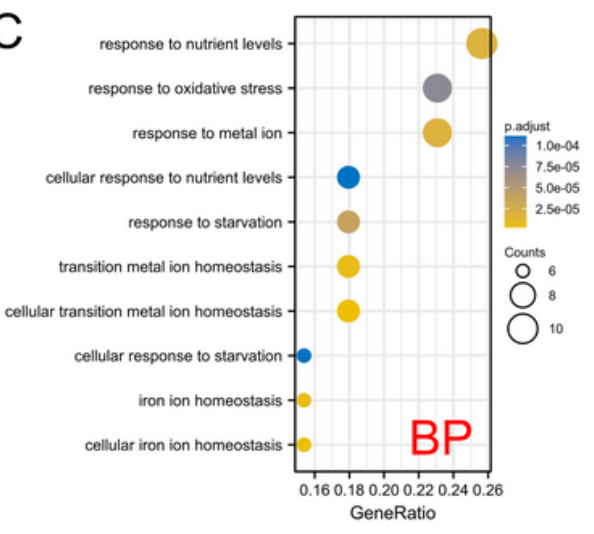

$E$

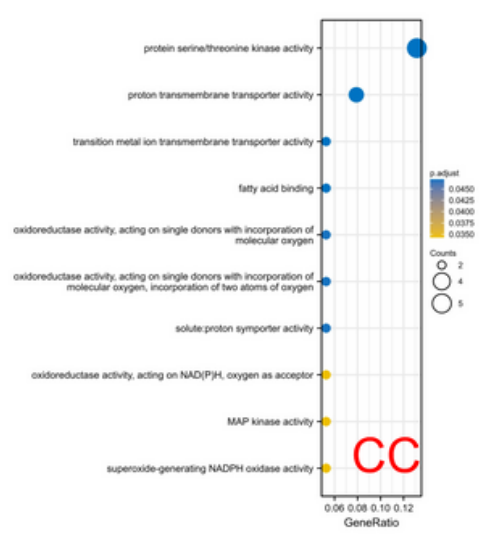

\section{Figure 8}

Analysis of the potential functions of 3 ferroptosis and iron-metabolism-related IncRNAs (FIRLs). (A) Regulation network diagram of the 49 upstream coding FIRGs of 3 FIRLs. (B) KEGG enrichment analysis of 49 upstream FIRGs. (C-E) GO enrichment analysis of 49 upstream FIRGs. 
A

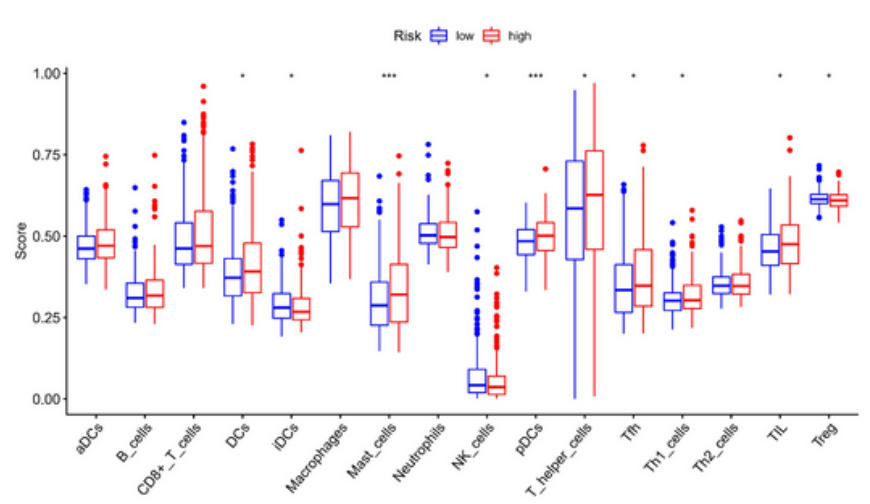

C

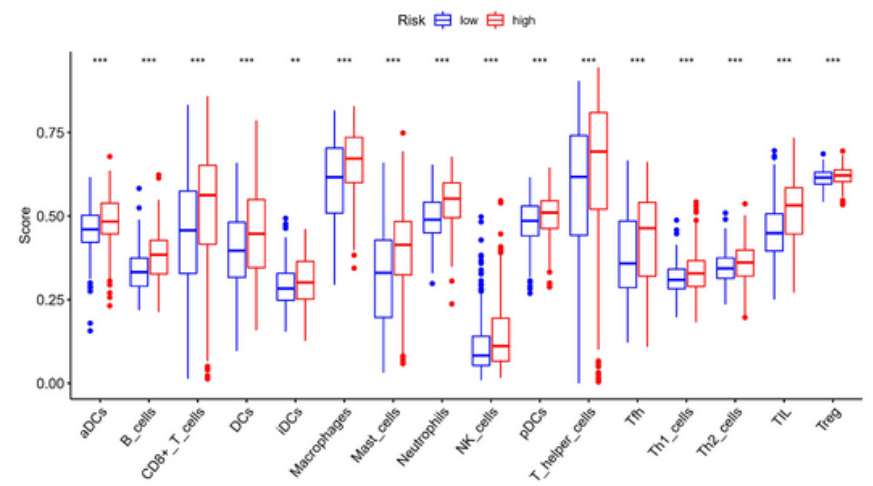

B

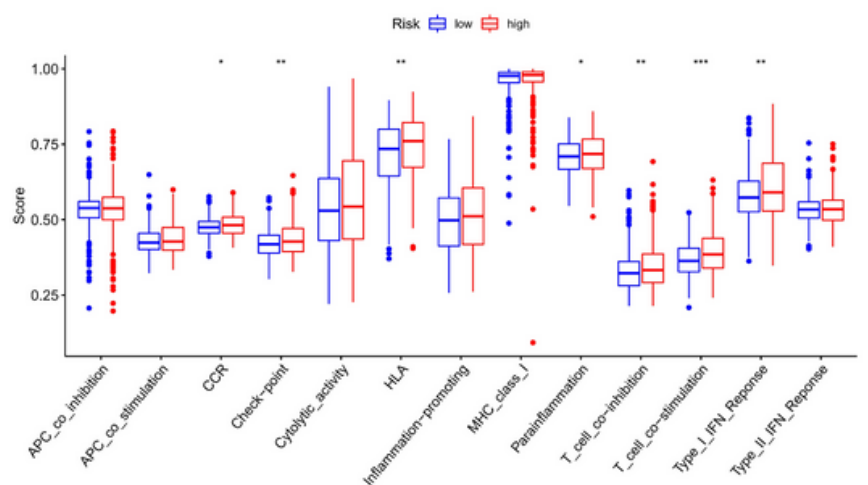

D

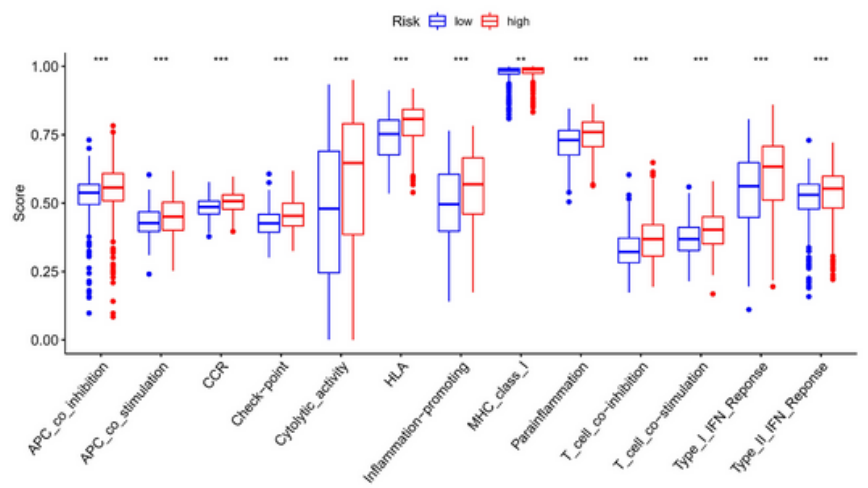

Figure 9

Comparison of the ssGSEA marks between the high-risk and low-risk groups. The scores of 16 immune cells $(A, C)$ and 13 immune-associated functions (B,D) are shown in boxplots. DCs, dendritic cells; iDCs, immature DCs; pDCs, plasmacytoid dendritic cells; TIL, tumor infiltrating lymphocyte; CCR, cytokinecytokine receptor; and APC, antigen presenting cells. Adjusted P values are displayed as: ns, not significant; ${ }^{*} \mathrm{P}<0.05 ; * \star \mathrm{P}<0.01 ; * \star * \mathrm{P}<0.001$. 


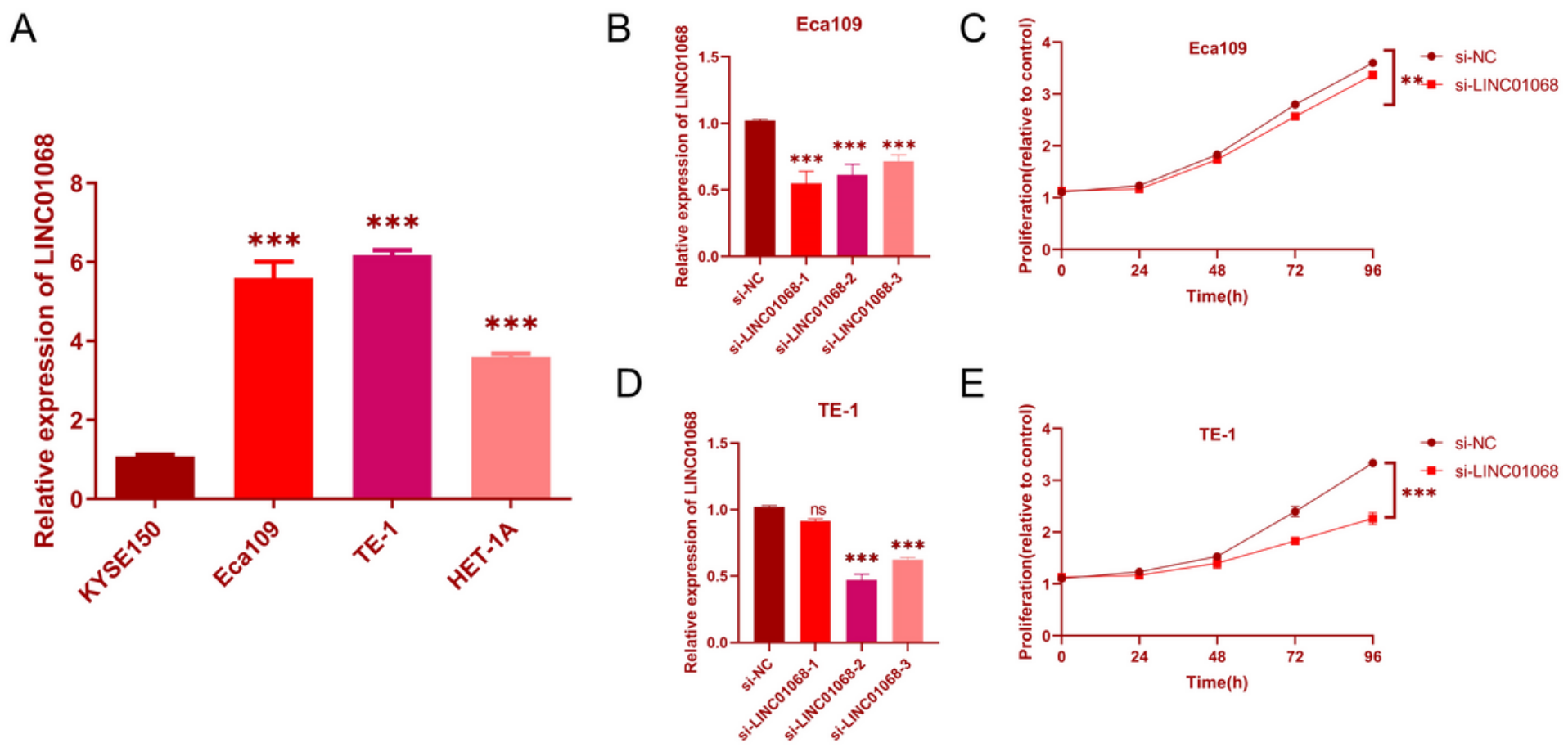

Figure 10

Effects of inhibiting the expression of LINC01068 on cell proliferation. (A) The upregulation of LINC01068 was displayed in Eca109, TE-1, and HET-1A cells compared to KYSE-150 based on qRT-PCR. (B, D) The expression of LINC01068 was downregulated in KYSE-150 and Eca109 cells by si-RNAs. (C, E) TE-1 and Eca109 cell proliferation after measuring anti-LINC01068 siRNA transfection with CCK-8 assays.

\section{Supplementary Files}

This is a list of supplementary files associated with this preprint. Click to download.

- TableS1.docx

- Tables2.xlsx 